(2)

\title{
Etiopathology and neurobiology of obsessive- compulsive disorder: focus on biological rhythms and chronotherapy
}

This article was published in the following Dove Press journal:

ChronoPhysiology and Therapy

20 May 2016

Number of times this article has been viewed

\author{
Rébecca Robillard ${ }^{1,2}$ \\ Addo Boafo 1,3,4 \\ 'University of Ottawa Institute of \\ Mental Health Research, ${ }^{2}$ School of \\ Psychology, University of Ottawa, \\ ${ }^{3}$ Department of Psychiatry, Children's \\ Hospital of Eastern Ontario, \\ ${ }^{4}$ Department of Psychiatry, University \\ of Ottawa Medical School, Ottawa, \\ ON, Canada
}

\begin{abstract}
This review examines biological rhythms in persons with obsessive-compulsive disorder (OCD) and their potential relevance to the pathophysiology and treatment of this disorder. In some cases of OCD, the expression of affective, cognitive, and behavioral symptoms may be influenced by circadian and seasonal rhythms, and this could possibly interact with other neurophysiological factors. Further work is required to characterize circadian profiles linked to OCD, but findings thus far highlighted delays in both the sleep-wake cycle and melatonin secretion, as well as reduced circadian rhythmicity of body temperature. It is proposed that these changes in behavioral and endogenous rhythms may increase one's vulnerability to obsessivecompulsive symptoms. Accordingly, obsessive-compulsive symptoms appear to be more severe in individuals with lower circadian amplitude and often worsen in the afternoon and evening. An increasing number of studies reported encouraging outcomes following the integration of sleep and circadian-based treatments in the management of OCD. There is a need for larger controlled trials evaluating the efficacy of chronotherapies in the context of OCD.
\end{abstract}

Keywords: chronobiology, circadian rhythms, sleep-wake cycle

\section{Introduction}

Obsessive-compulsive disorder (OCD) is a complex and heterogeneous neuropsychiatric condition. An increasing body of research indicates that the constellation of affective, cognitive, and behavioral aspects of OCD may interact with sleep and chronobiological processes, and this is generating valuable insights for new therapeutic applications. The present review summarizes pathophysiological and etiological features of OCD and examines how they may relate to abnormalities in sleep and biological rhythms. Implications for the management of obsessive-compulsive (OC) symptoms and the potential use of circadian-based therapeutic strategies are also discussed.

\section{Overview of OCD clinical features}

OCD is characterized by persistent and intrusive thoughts, images, and urges (obsessions; eg, contamination, pathological doubt, somatic concerns, symmetry) and repetitive behaviors or mental acts generally performed under rigid sets of rules in response to obsessions (compulsions; eg, checking, washing, counting). Typically, patients acknowledge the illogical or exaggerated nature of these thoughts and behaviors but struggle to overcome obsessions and resist engaging in compulsions. In the context of OCD, obsessions or compulsions are time-consuming (eg, take $>1$ hour per day) or 
cause clinically significant distress or impairment in social, occupational, or other important areas of functioning. ${ }^{1-4}$ Accordingly, OCD is among the top ten medical illnesses most likely to cause disability. ${ }^{5}$

Previously classified as an anxiety disorder, OCD has been reclassified in the fifth edition of the Diagnostic and Statistical Manual of Mental Disorders within "Obsessive Compulsive and Related Disorders". This new group of disorders also encompasses body dysmorphic disorder, hoarding disorder, trichotillomania, and excoriation disorder. This reclassification was notably based on symptom similarity, course of illness, biomarkers, and treatment response. ${ }^{6}$ These disorders were deemed to require a somewhat distinctive conceptual, assessment, and interventional approach compared to anxiety disorders. Indeed, an increasing body of research highlights distinct neurophysiological features differentiating obsessions and compulsions from anxiety symptoms. Similarly, while anxiety disorders are often accompanied by sleep and circadian disruptions, the distinct constellation of affective, cognitive, and behavioral aspects of OCD may interact with sleep and chronobiological processes in a unique manner.

\section{The chronobiological profile of OCD Abnormalities in endogenous rhythms}

The master biological clock in the suprachiasmatic nucleus (SCN) regulates 24-hour variations in numerous physiological functions. These endogenous rhythms influence behaviors, cognition, and subjective mood, and building evidence suggest that they may interact with psychopathological processes. Thus far, chronobiology studies in people with OCD reported possible abnormalities in melatonin, cortisol, prolactin, and temperature rhythms.

In a seminal study, drug-free adults with OCD were found to have lower mesor and amplitude of the circadian rhythm of melatonin compared to healthy controls, with prominent reductions in melatonin secretion in the first portion of the night, and an acrophase peaking with a delay of $\sim 2$ hours. $^{7}$ Two other studies failed to show significant abnormalities in the circadian pattern of melatonin, ${ }^{8,9}$ but these results may have been affected by small sample size and important methodological limitations, such as poor control of light exposure. In addition to melatonin, cortisol and prolactin are of particular interest in the chronobiology of OCD, notably because of their sensitivity to stress.

While OCD is linked to an overall increase in cortisol levels, ${ }^{7,8,10}$ the few studies which assessed cortisol variations across the 24-hour period observed no marked difference in circadian rhythmicity, amplitude, or phase between adults with a diagnosis of OCD and healthy controls. ${ }^{7-9}$ However, careful examination of the circadian curves reported in these studies suggests that people with OCD have much greater temporal instability and interindividual variability in nocturnal cortisol levels than healthy controls. In addition, a case study in a 29-year-old man with OCD reported elevated cortisol levels in the evening and early morning in comparison to healthy men in their twenties. ${ }^{11}$ Children and adolescents with OCD were found to have elevated cortisol levels in the early morning but not later in the morning nor in the evening. ${ }^{12}$ Thus, elevated cortisol secretion in the context of OCD may be subjected to circadian modulation in some individuals, and this may be especially prominent during youth and young adulthood.

Compared to cortisol, the chronobiology of prolactin has received less attention. In healthy, well-entrained individuals, prolactin typically undergoes ultradian and circadian variations, with secretion pulses at a period of $\sim 95$ minutes and a progressive increase during the night. ${ }^{13}$ In individuals with OCD, the pattern of prolactin rhythms was found to be altered, with increased levels prior to and after sleep initiation and a subsequent decrease across the night. ${ }^{7}$

To our knowledge, only one study has assessed 24-hour variations in body temperature in OCD. This study was based on axillary temperature measured at 3-hour intervals in eight adults with OCD and eight healthy controls. While this is yet to be replicated in larger samples, results suggested that individuals with OCD have poorer circadian rhythmicity than healthy controls, without any clear abnormality in circadian timing or mesor. ${ }^{9}$

Overall, in people with OCD, there are indications of flatter and delayed melatonin rhythms, possible increases in nocturnal cortisol and prolactin levels, and less-organized temperature rhythms. While the pathophysiological mechanisms underlying these circadian abnormalities remain unknown, these abnormalities may be indicative of weaker and partially delayed circadian signals. Importantly, there appears to be a progressive association between some circadian abnormalities and the severity of OC symptoms. For instance, worse OC symptoms as rated on the Yale-Brown Obsessive Compulsive Scale correlate with lower melatonin amplitude and mesor, lower circadian amplitude of prolactin, and lower cortisol mesor. ${ }^{7}$ Further investigations are warranted, notably to clarify how specific these circadian disruptions are to OCD compared to other mental disorders.

\section{Sleep-wake cycle}

Recent studies linked OC symptoms severity to sleep disturbances. ${ }^{14-16}$ Objective findings consistently highlighted 
reductions in sleep duration and poor sleep consolidation, ${ }^{15}$ and increasing evidence indicate that sleep initiation difficulties and delayed sleep phase disorder (DSPD) are also common in OCD. Studies based on self-report, nursing notes, and medical records estimated that $17 \%-42 \%$ of inpatients admitted to specialized OCD clinic met criteria for DSPD and reported that this subgroup of patients tended to have more severe OC symptoms. ${ }^{17,18}$ In another study based on actigraphic sleep monitoring, delayed sleep profiles were found in $25 \%$ of inpatients with OCD. ${ }^{19}$ Albeit variable, these rates are considerably higher than the $0.2 \%-10 \%$ reported in the general population. ${ }^{20}$

Such observations have also been reported outside of formal clinical settings. In a sample of university students, OC symptoms were significantly higher in individuals with delayed sleep schedules than in those with earlier sleep schedules. ${ }^{21}$ In fact, the proportion of students with clinically meaningful levels of OC symptoms was almost double in the subgroup with delayed sleep. Furthermore, in a small community sample, individuals with a delayed sleep profile endorsed worse anxiety and OC symptoms and were more distressed by OC symptoms than those with an earlier sleep schedule. ${ }^{22}$ In these two studies, worse OC symptoms were significantly correlated with later bedtimes, but findings conflicted as to whether this association may be driven by underlying depressive states.

Disturbances of the sleep-wake cycle in persons with OCD are likely to be influenced by endogenous circadian rhythms. Specifically, abnormal nighttime endocrine patterns, such as elevated cortisol and reduced/delayed melatonin, may contribute to a physiological state less conducive to sleep initiation and maintenance. This may lead to delayed, shortened, and fragmented sleep, which may, in turn, worsen alterations in endogenous rhythms. Importantly, delayed sleep patterns could also possibly interact with the emergence of OCD symptoms in the evening. On the one hand, the urge to complete extended evening rituals can significantly delay bedtime, and obsessive thoughts can lengthen sleep onset latency. On the other hand, increased homeostatic pressure, which may result from sleep curtailing (eg, when one has a delayed sleep profile and attempts to follow early morning school/work schedules), can increase cortisol sensitivity to stress and induce anxiety. ${ }^{23,24}$ Also, as will be covered in the following sections, circadian and homeostatic influences may exacerbate nighttime neurocognitive dysfunctions, and this could further increase one's difficulty to disengage from obsessive thoughts and resist/ terminate compulsive behaviors.

\section{Cyclical patterns of OC symptoms}

Animal studies suggest that cortisol and prolactin are more reactive to stress at the beginning of the biological night than at the beginning of the biological day. ${ }^{25,26}$ While this remains to be directly tested in humans, it may be postulated that OCD-related abnormalities in the circadian regulation of these hormones could be linked to an increased vulnerability to OC symptoms at night.

Indeed, case reports dating from as early as the beginning of the 20th century described circadian variations in the occurrence of compulsive ritual behaviors. ${ }^{27}$ Subsequent studies in persons with OCD did not report consistent diurnal patterns in OC symptoms monitored over 1-3 days. ${ }^{9,28,29}$ However, more recently, symptoms monitoring over an average of 7 days suggested that obsessions occur more frequently in the afternoon but revealed considerable inter- and intraindividual differences. ${ }^{30}$ In addition, a few case reports highlighted increased compulsions late at night, with worse difficulties to dismiss intrusive thoughts and resist the urge to engage in behaviors with excessive perfectionism..$^{31,32}$ These daily rhythms in OC symptoms may possibly be potentiated by circadian variations in parallel symptoms, such as general anxiety, which has also been observed to worsen in the afternoon or evening. ${ }^{33}$

OC symptoms have been proposed to relate to cognitive inhibition and working memory dysfunctions, ${ }^{34}$ two components of executive functions which are sensitive to interactions between homeostatic and circadian processes. ${ }^{35,36}$ However, sleep and circadian influences on the cognitive aspects of OC symptoms have yet to be directly investigated. Functional magnetic resonance imaging indicates frontoparietal hyperactivations during an n-back task in OCD patients compared to healthy controls, a phenomenon proposed to reflect compensatory mechanisms elicited by working memory difficulties. ${ }^{37}$ Interestingly, in healthy persons, the recruitment of frontal resources by a similar n-back task has been shown to vary according to homeostatic pressure and circadian phase, and these changes take different forms depending on a polymorphism linked to circadian misalignment and sleep loss vulnerability. ${ }^{38}$ The distribution of this polymorphism in OCD has yet to be investigated. Nevertheless, it may be hypothesized that the common occurrence of delayed sleep onset in persons with OCD, in combination with a possibly reduced circadian signal, may limit the recruitment of frontal areas to compensate for cognitive difficulties, especially in the evening and nighttime when homeostatic pressure is high.

In conjunction with cognitive and neurobehavioral factors, daily variations in OC symptoms may also be influenced 
by the accumulated exposure to potential stressors across the day, as well as social rhythms and other environmental factors. ${ }^{29,30}$ For instance, occupational activities and frequent exposure to strangers or work/school colleagues may increase the pressure to repress compulsive behaviors during the daytime ${ }^{21}$ or even act as distracters from obsessive thoughts. Conversely, obsessions and compulsions involving other family members, or various aspects of one's living environment, are likely to emerge after working/school hours. In addition, greater occurrences of OC symptoms at night are likely to increase behavioral/cognitive activation and light exposure $^{15,32}$ at a sensitive circadian phase, and this may contribute to further delays in circadian rhythms and the sleep-wake cycle.

In brief, findings reported so far seem to indicate that obsessions may intensify in the afternoon/evening, and that inhibiting compulsive enactment appears to be markedly difficult during late evening/early night. These circadian patterns of OC symptoms may be modulated by endogenous biological rhythms, variations in cognitive and neuronal resources, the daily rhythms of socio-occupational life, and environmental factors. Nevertheless, further empirical studies are required to decipher cyclic patterns in OC symptomatology and their underlying physiological, cognitive, and behavioral mechanisms.

\section{Chronobiology and the etiopathology of OCD}

Hypothalamic-pituitary-adrenal axis hyperactivity has long been proposed to contribute to both cortisol and melatonin abnormalities in OCD. ${ }^{8}$ In light of recent neurobiological findings, additional interactions between OCD pathophysiology and abnormalities in the sleep-wake cycle and biological rhythms can be postulated. An overview of these interactions is provided in Figure 1.

\section{Neurobiological models}

At the neurotransmission level, the antiobsessional effects of selective serotonin reuptake inhibitors (SSRIs) and their common therapeutic effects in OCD led to the hypothesis of serotonergic dysfunctions in OCD. ${ }^{39}$ More recently, abnormalities in serotonin transporter availability in the thalamus, midbrain, and brain stem have been reported in persons with OCD. ${ }^{40,41}$ Dysfunctions in noradrenergic system have also been postulated. ${ }^{42}$ Both serotonin and noradrenalin follow a circadian rhythm and are involved in melatonin synthesis. It has thus been proposed that deregulation in melatonin secretion in persons with OCD may relate to increased noradrenergic and reduced serotonergic tone., Since prolactin, and in some cases cortisol, sensitivity to serotonin appears to be blunted in some persons with $\mathrm{OCD},{ }^{43}$ circadian dysregulation in these hormones may also relate to serotonergic dysfunctions. In addition, chronic sleep loss resulting from delayed bedtime may possibly further desensitize 5-hydroxytryptamine (5-HT) 1 receptors. ${ }^{44}$

From a structural perspective, OCD is notably characterized by smaller orbitofrontal, anterior cingulate, and temporolimbic cortices (ie, structures heavily involved in affective regulation), and smaller dorsomedial, dorsolateral, ventrolateral, and frontopolar prefrontal cortices (ie, structures heavily involved in executive functions). ${ }^{45}$ Functional studies in OCD indicated increased activations in the corticostriato-thalamo-cortical circuit, particularly in the caudate, putamen, anterior thalamus, and orbitofrontal cortex, ${ }^{46-52}$ and also in the amygdala. ${ }^{53}$ In OCD, these structures are hyperactivated by OC symptom provocation, and this hyperactivation resolves in parallel with treatment-related improvements. ${ }^{54-56}$ These abnormalities in orbitofrontal-subcortical circuits are thought to cause executive dysfunctions leading to the emergence of OC behaviors, which, in turn, may reinforce orbitofronto-subcortical abnormalities. ${ }^{57}$ In addition, observations of reduced responses of the nucleus accumbens during reward anticipation ${ }^{58}$ and attenuated prefrontal activations during loss anticipation ${ }^{59}$ have reinforced the notion that functional alterations in key components of the corticostriato-thalamo-cortical circuit may be involved in abnormal reward processing in OCD.

Circadian variations in reward-related behaviors (peaking in the afternoon/evening ${ }^{60,61}$ ) are thought to be partially regulated by the master biological clock in the $\mathrm{SCN}$ via dopaminergic signaling in the mesolimbic pathway. ${ }^{62}$ Recently, it has been proposed that stress-related alterations in subordinate biological clocks, notably in the reward network, may contribute to a desynchronization between central and peripheral circadian signals. ${ }^{63}$ Others have hypothesized that circadian misalignment may induce alterations in executive functioning, behavioral inhibition, and reward-related regulation, ${ }^{64,65}$ all factors highly involved in OCD symptomatology.

Sleep curtailing related to delayed sleep profile and chronic sleep difficulties may also worsen the neurobiological profile of OCD. Sleep loss is known to lead to reduced metabolism in the striatum, thalamus, and frontal cortex, ${ }^{66}$ and to alter executive functions relevant to OCD. ${ }^{67}$ Also, partial and full sleep deprivation increase appetitive-related/ pleasure-related activations in the putamen, thalamus, and orbitofrontal cortex. ${ }^{68,69}$ OCD abnormalities in the reward 


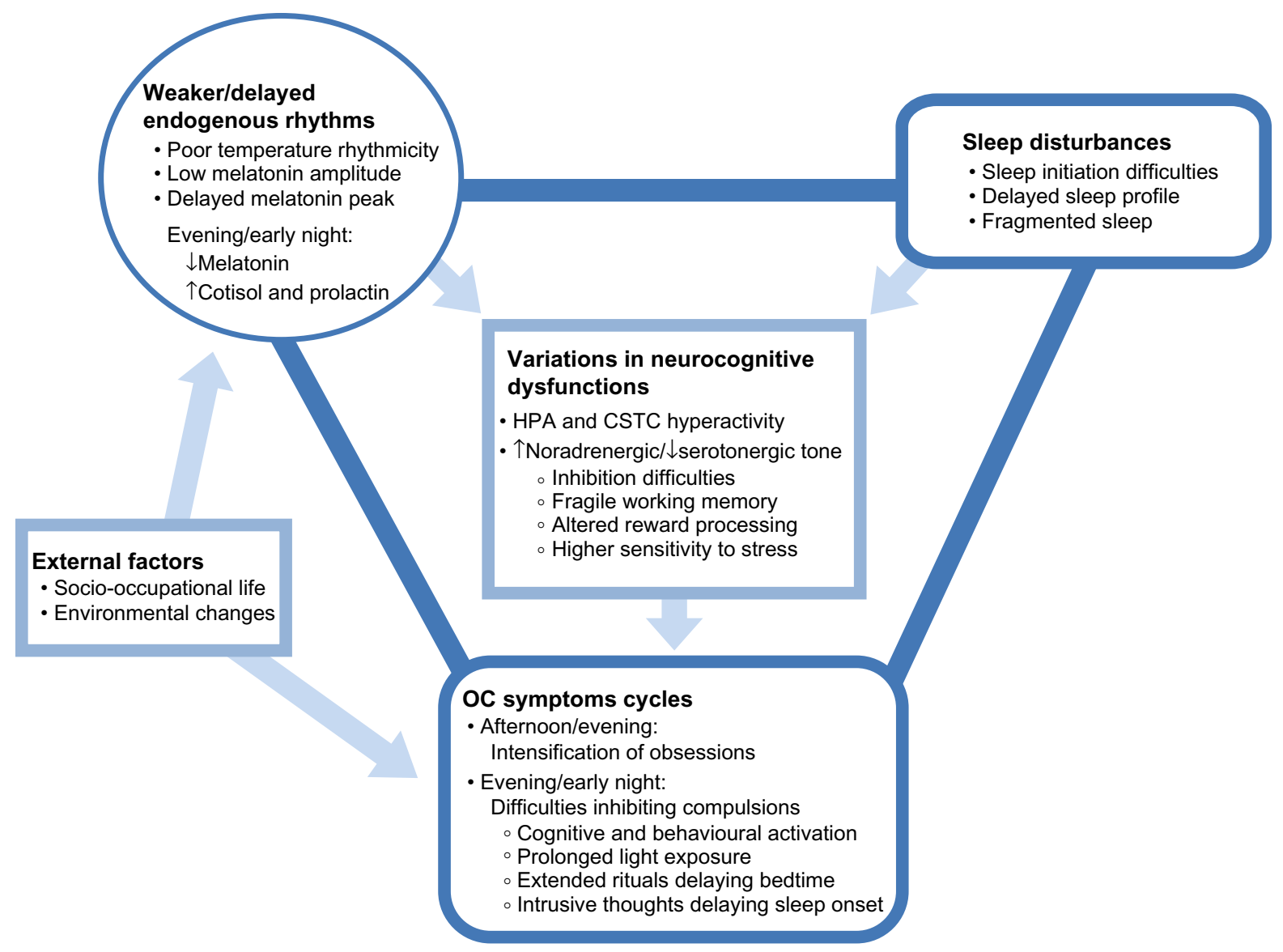

Figure I Proposed interactions between OCD pathophysiology, the sleep-wake cycle, and biological rhythms.

Notes: $\uparrow$, increase; $\downarrow$, decrease.

Abbreviations: OCD, obsessive-compulsive disorder; HPA, hypothalamic-pituitary-adrenal; CSTC, cortico-striato-thalamo-cortical; OC, obsessive-compulsive.

network may thus interact with changes driven by increased homeostatic pressure. The relevance of these theoretical frameworks for OCD pathophysiology has yet to be formally tested.

\section{Genetic and molecular factors}

Twin studies show a high heritable component to OCD, with concordance rates of $80 \%-87 \%$ in monozygotic twins and $47 \%-50 \%$ in dizygotic twins. ${ }^{70}$ There is a fourfold increased risk of OCD among relatives of probands. ${ }^{71}$ Over the last decade, $>80$ OCD candidate genes have been identified. ${ }^{72}$ Yet, to our knowledge, no study has directly investigated the associations between OCD and the "clock" genes involved in the transcriptional-translational autoregulatory loop regulating the circadian clock. However, there is evidence that common genetic factors may be linked to some of the neurofunctional, behavioral, and chronobiological characteristics of OCD.

The expression of "clock" genes in components of the reward network known to be altered in OCD (eg, prefron- tal cortex, amygdala, and nucleus accumbens) follows a strong circadian pattern. ${ }^{73}$ In addition, later sleep schedules and polymorphisms of the PER2 gene predict lower reward-related activations in the prefrontal cortex, which may reflect reduced behavioral self-monitoring.$^{74}$ Considering the behavioral and neurophysiological abnormalities of reward processing in OCD, these results call for direct investigations of PER2 polymorphisms in people with OCD.

In addition, the met allele of the $B D N F$ gene Val66Met polymorphism, which is characterized by reduced BDNF activity, has been linked to executive dysfunctions in OCD. ${ }^{75}$ Interestingly, this met allele has also been associated with higher spontaneous cortisol secretion in the evening, but not in the morning, ${ }^{76}$ increased cortisol response to stress, ${ }^{77}$ and lower sleep intensity in the first portion of the night. ${ }^{78}$

Recent advances in epigenetics have shown that alterations in sleep duration and timing attenuate the circadian patterns of gene expression. ${ }^{79,80}$ From this perspective, sleep fragmentation and delayed sleep profiles related to mental disorders such as OCD may thus disturb the molecular clock 
and actively contribute to reduce circadian rhythmicity and amplitude, two factors associated with worsening of $\mathrm{OC}$ symptoms severity. While this hypothesis remains speculative, the cross-road between sleep/circadian epigenetics and mental disorders yields a promising new field of investigation.

\section{Therapeutic implications}

The potential involvement of sleep and circadian disruptions in the etiopathology of OCD calls for the application of chronotherapeutics, or treatment strategies targeting the biological clock, in the management of OCD. The following sections review the main therapies classically used for OCD and new insights about chronotherapeutic applications.

\section{Current OCD therapies}

\section{Psychotherapies}

Cognitive behavior therapy (CBT), which typically includes exposure and response prevention and cognitive therapy, is recognized as the first-line evidence-based psychological treatment for OCD. ${ }^{81,82}$ Exposure and response prevention involves developing a hierarchy of triggering thoughts, images, objects, and situations, which are then ordered from least fear producing to most fear producing. Then, the patient is systematically guided through exposure to situations on the hierarchy long enough to reach habituation. The response prevention aspect of this intervention includes having the patients resist engaging in their compulsions. Cognitive therapy is based on the identification and modification of dysfunctional appraisals of compulsions and symptom-related beliefs in order to impact problematic behavior. Some of the challenges of CBT for OCD include the limited number of qualified therapists, treatment refusal, dropout rates (rising up to $30 \%$ ), common residual symptoms, and lack of sustained improvements in $\sim 45 \%$ of cases. ${ }^{83}$

\section{Pharmacotherapies}

From a pharmacological perspective, SSRIs, such as fluoxetine, sertraline, and fluvoxamine, are the recommended first-line treatment for OCD. ${ }^{84,85}$ It may take up to 12 weeks of pharmacotherapy to produce a significant change in symptoms, and response rates are estimated to range between $40 \%$ and $60 \%{ }^{86-89}$ Second-line options include clomipramine (a tricyclic antidepressant), venlafaxine, and mirtazapine (selective noradrenergic reuptake inhibitors).

Some of the OCD patients who are nonresponsive to pharmacotherapy and behavior therapy have been shown to benefit from other treatment options. These include adding a dopamine blocker, such as risperidone or aripiprazole, in low doses to an SSRI. Also, recent evidence also shows that some of the refractory OCD patients benefit from medications related to the glutamatergic system such as D-cycloserine, riluzole, and memantine. ${ }^{90}$ Additionally, some treatmentresistant patients have responded positively to stereotactic surgery targeting structures and pathways within the basal ganglia-thalamo-cortical circuits, ${ }^{91}$ as well as treatment by high-frequency stimulation of parts of the putative OCD neuro-circuitry, such as the anterior limb of the internal capsule, the nucleus accumbens, the anterior caudate nucleus, and the anterior ventral part of the subthalamic nucleus. ${ }^{92}$ Other common augmentation options include the use of some second-generation antipsychotics (low-dose risperidone or aripiprazole), D-amphetamine, topiramate, or ondansetron.

To summarize, CBT and SSRIs are probably the most common therapeutic strategies used with patients with OCD. However, their effectiveness is limited by relatively late therapeutic responses, common adherence problems, and frequent residual symptoms. There is thus a need for novel interventions for OCD.

\section{Potential applications of chronotherapies}

If the pathophysiology of OCD is linked to, or exacerbated by, circadian dysfunctions, interventions aiming to restore the circadian clock may have positive effects on OC symptoms. In addition, since the cognitive, biological, and anxiogenic consequences of disturbed sleep and circadian rhythms may impinge on therapeutic response to classical interventions for OCD, chronotherapeutics may also be relevant adjunctive strategies.

\section{Phototherapy}

The potential relevance of phototherapy in OCD has been highlighted in a few reviews, ${ }^{93,94}$ but empirical data on this topic are scarce. Two case studies in patients with seasonal affective disorder and OCD peaking in fall and winter reported clinical improvements in both mood and OC symptoms after 5-12 days of phototherapy $(10,000$ lux for 30 minutes or 3,000 lux in the morning for 2 hours). ${ }^{95,96}$ In one case, the reintroduction of phototherapy following a subsequent relapse again led to marked improvements in both mood and OCD symptoms, ${ }^{95}$ and in the other case, there was no relapse in the next few months after phototherapy.

Seasonal changes in OC symptoms and mood difficulties have been reported in $21 \%$ and $53 \%$ of persons with OCD, respectively. ${ }^{97}$ In most cases, these changes were characterized by worse symptoms during winter and some degree of improvement during summer. The use of phototherapy to treat seasonal affective disorder has now reached expert 
consensus $^{98,99}$ and is increasingly implemented in clinical practice. There is a need to determine whether response to light interventions in people with OCD is modulated by seasonal variations in OC symptoms.

In addition to its direct chronobiotic effects, which may alleviate dysfunctions of the biological clock, the therapeutic effects of light in people with OCD could also possibly operate via the serotonergic system. There is a high density of 5-HT transporters in the SCN, especially in regions primarily involved in light-induced circadian phase shifts. ${ }^{100}$ Light exposure increases 5-HT concentrations in the pineal gland, and this is thought to play a role in the phase-shifting effects of light on melatonin and the sleep-wake cycle. ${ }^{101}$ In support of this, SSRIs administered to diurnal animals in the morning induced a phase advance of the activity-rest cycle. ${ }^{102}$

On the one hand, light exposure facilitates phase advances induced by 5 -HT agonists and SSRIs, ${ }^{103,104}$ and on the other hand, SSRIs potentiate light-induced phase advances. ${ }^{102}$ Therefore, a bidirectional relationship between light and the serotonergic system may be postulated with: 1) daily light exposure patterns affecting 5-HT regulation; and 2) 5-HT abnormalities modulating one's sensitivity to light. Controlled light exposure may thus be a promising therapeutic tool to attenuate serotonergic dysfunctions in $\mathrm{OCD}$, potentiate the efficacy of SSRIs commonly used to manage OC symptoms, and realign the sleep-wake cycle in OCD patients with delayed sleep profiles.

\section{Melatonin agents}

Agomelatine is a new antidepressant acting as a melatonin agonist at MT1 and MT2 receptors and as a selective serotonin antagonist at 5-HT2C receptors. This compound has rapid sleep-enhancing effects and chronobiotic properties, which mimic the effects of exogenous melatonin on the circadian system, allowing the realignment and strengthening of biological rhythms. ${ }^{105,106}$ Due to its unique pharmacological profile and its anxiolytic properties, ${ }^{107}$ agomelatine is increasingly considered as a potential adjunctive or alternative to standard OCD treatments. ${ }^{108}$

When added to classical pharmacological agents used for OCD (eg, escitalopram, venlafaxine, clomipramine, risperidone, and/or aripiprazole), agomelatine has been found to improve OC symptoms in several cases of treatment-resistant OCD. ${ }^{109-111}$ In some cases, this led to full remission after $\sim 3$ months of treatment, ${ }^{109}$ and in reports with longitudinal monitoring, clinical improvements were often sustained over several months. ${ }^{110,111}$ A multiple case study reported considerable clinical improvements in three out of six cases of treatment-resistant OCD. ${ }^{112}$ Interestingly, two of the three individuals who responded to agomelatine initially presented with sleep disruptions. In these two cases, sleep was found to improve alongside OC symptoms. To date, the strongest empirical support comes from an openlabel study conducted in 12 persons with OCD who did not respond to SSRIs. ${ }^{113}$ Significant reductions in OC symptoms were found after adjunctive agomelatine treatment. Importantly, only mild side effects (ie, nausea, headache, dizziness, and somnolence) were reported, suggesting that agomelatine is well tolerated in individuals with treatmentresistant OCD.

While most studies have thus far assessed the efficacy of agomelatine in combination with other classical pharmacological treatment for OCD, there are some preliminary indications supporting monotherapy with agomelatine. A young woman with OCD who gained little benefits with classical pharmacotherapy and experienced marked side effects underwent a full and sustained remission from OCD after $\sim 2$ months of treatment with agomelatine. ${ }^{114}$ Of note, this patient had sleeping difficulties related to intrusive thoughts about bedsheet contamination, and the first noticeable clinical improvements ( 2 weeks after the introduction of agomelatine) were sleep improvements. Hence, this supports the hypothesis that sleep restoration may contribute to subsequent OCD symptom reductions.

Agomelatine may also have beneficial effects in disorders related to OCD. For instance, in a young woman with excoriation disorder who did not respond to 25 days of administration of sertraline and quetiapine, agomelatine reduced skinpicking behavior, and this clinical improvement persisted after cessation of sertraline and quetiapine. ${ }^{15}$ Interestingly, it has also recently been proposed that agomelatine may be especially effective in a subgroup of patients with OCD who also present bipolar traits. ${ }^{108}$

While the mechanisms underlying its potential antiobsessive and anticompulsive effects remain speculative, agomelatine interacts with key neurotransmitters involved in the pathophysiology of OCD. For instance, animal studies demonstrated that this compound enhances the levels of dopamine and noradrenaline in the frontal cortex ${ }^{116}$ and that its synergistic effects on both melatonin and 5-HT receptors attenuate glutamate release following stress exposure. ${ }^{117}$ This may contribute to normalize circadian abnormalities linked to OCD and realign the sleep-wake cycle in those with delayed sleep profiles. We still need to assess whether other melatonin agonists (without influence on the 5-HT system) 
or exogenous melatonin supplementation may also reduce OC symptoms in some patients.

\section{Behavioral circadian approaches}

In early DSPD case reports, successful realignment of the sleep-wake cycle through behavioral phase advance led to enhanced psychological functioning. ${ }^{118}$ In the context of OCD with delayed sleep profiles, realigning the sleep-wake cycle may enhance psychological well-being and reduce some of the cognitive difficulties worsening nighttime compulsions. This could potentiate the effects of classical psychotherapies for OCD. Also, assisting patients in understanding and anticipating potential circadian variations in the emergence of OC symptoms may increase empowerment and maximize cognitive and affective resources for better symptoms management by the patients themselves. ${ }^{30}$

One case study reported successful outcomes following a combination of agomelatine and behavioral interventions targeting circadian realignment. ${ }^{32}$ The patient had long-standing religious and cleaning compulsions and did not respond to SSRIs and classical OCD-focused CBT. Initially, it was noted that she had a marked delay in her sleep-wake schedule, which was accompanied by fatigue, poor concentration, and worse difficulties inhibiting compulsions at nighttime. In fact, the later this patient initiated her nighttime rituals, the longer it took her to complete them. Her treatment was enriched with chronotherapeutic interventions; in addition to being started on agomelatine, she was guided to implement a progressive phase advance in the timing of her nighttime rituals, bedtime, and wake time. These behavioral changes were facilitated using motivational approaches and morning call-ins. This resulted in a decrease in her daily compulsion time from 8 to 2 hours and a marked reduction in the severity of her OC symptoms.

There is strong evidence for the efficacy of CBT-i, a form of CBT specifically designed for insomnia, ${ }^{119}$ with increasing applications in psychiatric populations. ${ }^{120}$ Some of the key components of CBT-i, such as paradoxical intention and relaxation techniques, are directly targeting sleep initiation difficulties and may thus be relevant tools for reducing sleep onset latency and phase advancing the sleep-wake cycle. There is currently no report on the usefulness of such techniques in OCD.

In addition to adjusting the timing of sleep, other circadian synchronizers ("zeitgeber") can be targeted by behavioral changes in more holistic approaches. OCD is often characterized by a greater sensitivity to stress. In line with the "social zeitgeber" theory, ${ }^{121}$ it has been proposed that life stressors may interfere with social routines, leading to altered circadian rhythms and facilitating the emergence of certain OCD profiles. ${ }^{112}$ In fact, OCD is often accompanied by social isolation, reduced time engaging in purposeful activities, and irregular meal times, all factors likely to tone down social synchronizers. Interpersonal and social rhythm therapy, a circadian-based intervention initially developed to treat bipolar disorder, aims to stabilize social circadian rhythms, establishing daily routines for sleep, wake, eating, and purposeful activities. ${ }^{122,123}$ Considering the common tendency to engage in compulsive behavior late in the day and the frequent comorbidity between OCD, DSPD, and bipolar disorders or cyclothymic traits, ${ }^{124-127}$ interpersonal and social rhythm therapy may provide useful tools to guide relevant behavioral changes in certain patients with OCD.

\section{Conclusion}

A range of circadian disruptions has been documented in persons with OCD, including reduced circadian rhythmicity of body temperature and delays in both the sleep-wake cycle and melatonin secretion. These abnormalities appear to correlate with worse symptom severity. It is worth noting that studies conducted to date were typically based on small numbers of participants and that there appears to be considerable interindividual variability across persons with OCD. OCD is often recognized as a heterogeneous illness, and this heterogeneity may also color the sleep and circadian profiles linked to OCD. As such, further investigations with larger sample sizes, better controlled circadian monitoring, and careful examination of potential subgroups of patients with distinct circadian profiles and clinical trajectories are required.

Importantly, OCD presents as a unique psychiatric illness in which biological, behavioral, and cognitive psychopathological processes may interact directly with circadian abnormalities. Evidence suggests that the symptomatology of OCD often follows cyclic patterns, and some of the pathogenetic mechanisms underlying this disorder may be sensitive to biological, social, and environmental rhythms.

Following promising initial reports, there is a need for better powered and well-controlled clinical trials to assess the efficacy of sleep and circadian-based interventions for the management of OCD. Rigorous consideration of age, comorbidities, genetic predispositions, response to classical OCD treatment, objective sleep and circadian profiling, and seasonal course of illness will be helpful to identify relevant factors predicting response to chronotherapies in patients with OCD.

\section{Acknowledgments}

Dr Robillard was supported by a fellowship from the Fonds de recherche du Québec - Santé. The authors thank 
Professor Joseph De Koninck for his helpful comments on the manuscript.

\section{Disclosure}

The authors report no conflicts of interest in this work.

\section{References}

1. Masellis M, Rector NA, Richter MA. Quality of life in OCD: differential impact of obsessions, compulsions, and depression comorbidity. Can J Psychiatry. 2003;48(2):72-77.

2. Koran LM, Thienemann ML, Davenport R. Quality of life for patients with obsessive-compulsive disorder. Am J Psychiatry. 1996;153(6):783-788.

3. Eisen JL, Mancebo MA, Pinto A, et al. Impact of obsessivecompulsive disorder on quality of life. Compr Psychiatry. 2006;47(4): 270-275.

4. American Psychiatric Association. Diagnostic and Statistical Manual of Mental Disorders. 5th ed. Arlington, VA: American Psychiatric Publishing; 2013.

5. Murray CJ, Lopez AD. The Global Burden of Disease: A Comprehensive Assessment of Mortality and Disability from Diseases, Injuries, and Risk Factors in 1990 and Projected to 2020. Cambridge, MA: Harvard University Press; 1996.

6. Stein DJ, Fineberg NA, Bienvenu OJ, et al. Should OCD be classified as an anxiety disorder in DSM-V? Depress Anxiety. 2010;27(6): 495-506.

7. Monteleone P, Catapano F, Del Buono G, Maj M. Circadian rhythms of melatonin, cortisol and prolactin in patients with obsessive-compulsive disorder. Acta Psychiatr Scand. 1994;89(6):411-415.

8. Catapano F, Monteleone P, Fuschino A, Maj M, Kemali D. Melatonin and cortisol secretion in patients with primary obsessive-compulsive disorder. Psychiatry Res. 1992;44(3):217-225.

9. Millet B, Touitou Y, Poirier MF, et al. Plasma melatonin and cortisol in patients with obsessive-compulsive disorder: relationship with axillary temperature, physical activity, and clinical symptoms. Biol Psychiatry. 1998;44(9):874-881

10. Gehris TL, Kathol RG, Black DW, Noyes R Jr. Urinary free cortisol levels in obsessive-compulsive disorder. Psychiatry Res. 1990;32(2): $151-158$.

11. Bigos KL, Folan MM, Jones MR, Haas GL, Kroboth FJ, Kroboth PD. Dysregulation of neurosteroids in obsessive compulsive disorder. $J$ Psychiatr Res. 2009;43(4):442-445.

12. Gustafsson PE, Gustafsson PA, Ivarsson T, Nelson N. Diurnal cortisol levels and cortisol response in youths with obsessive-compulsive disorder. Neuropsychobiology. 2008;57(1-2):14-21.

13. Veldhuis JD, Johnson ML. Operating characteristics of the hypothalamopituitary-gonadal axis in men: circadian, ultradian, and pulsatile release of prolactin and its temporal coupling with luteinizing hormone. J Clin Endocrinol Metab. 1988;67(1):116-123.

14. Cox RC, Olatunji BO. Sleep disturbance and obsessive-compulsive symptoms: results from the national comorbidity survey replication. J Psychiatr Res. 2016;75:41-45.

15. Paterson JL, Reynolds AC, Ferguson SA, Dawson D. Sleep and obsessivecompulsive disorder (OCD). Sleep Med Rev. 2013;17(6):465-474.

16. Timpano KR, Carbonella JY, Bernert RA, Schmidt NB. Obsessive compulsive symptoms and sleep difficulties: exploring the unique relationship between insomnia and obsessions. J Psychiatr Res. 2014;57:101-107.

17. Mukhopadhyay S, Fineberg NA, Drummond LM, et al. Delayed sleep phase in severe obsessive-compulsive disorder: a systematic case-report survey. CNS Spectr. 2008;13(5):406-413.

18. Turner J, Drummond LM, Mukhopadhyay S, et al. A prospective study of delayed sleep phase syndrome in patients with severe resistant obsessivecompulsive disorder. World Psychiatry. 2007;6(2):108-111.
19. Drummond LM, Wulff K, Rani RS, et al. How should we measure delayed sleep phase shift in severe, refractory obsessive-compulsive disorder? Int J Psychiatry Clin Pract. 2012;16(4):268-276.

20. Lack LC, Wright HR, Bootzin RR. Delayed sleep-phase disorder. Sleep Med Clin. 2009;4(2):229-239.

21. Coles ME, Schubert JR, Sharkey KM. Delayed bedtimes and obsessivecompulsive symptoms. Behav Sleep Med. 2012;10(4):258-265.

22. Schubert JR, Coles ME. Obsessive-compulsive symptoms and characteristics in individuals with delayed sleep phase disorder. J Nerv Ment Dis. 2013;201(10):877-884.

23. Minkel J, Moreta M, Muto J, et al. Sleep deprivation potentiates HPA axis stress reactivity in healthy adults. Health Psychol. 2014;33(11):1430-1434.

24. Babson KA, Trainor CD, Feldner MT, Blumenthal H. A test of the effects of acute sleep deprivation on general and specific self-reported anxiety and depressive symptoms: an experimental extension. J Behav Ther Exp Psychiatry. 2010;41(3):297-303.

25. Dunn J, Scheving L, Millet P. Circadian variation in stress-evoked increases in plasma corticosterone. Am J Physiol. 1972;223(2): 402-406.

26. Kant GJ, Mougey EH, Meyerhoff JL. Diurnal variation in neuroendocrine response to stress in rats: plasma ACTH, beta-endorphin, beta$\mathrm{LPH}$, corticosterone, prolactin and pituitary cyclic AMP responses. Neuroendocrinology. 1986;43(3):383-390.

27. Janet P. Les obsessions et la psychasthenie [Obsessions and Psychasthenia]. Paris: Bailliere; 1908. French.

28. Millet B, Touitou Y, Poirier MF, et al. Obsessive-compulsive disorder: evaluation of clinical and biological circadian parameters during fluoxetine treatment. Psychopharmacology. 1999;146(3): 268-274.

29. Herman S, Koran LM. In vivo measurement of obsessive-compulsive disorder symptoms using palmtop computers. Comput Hum Behav. 1998;14(3):449-462.

30. Nota JA, Gibb BE, Coles ME. Obsessions and time of day: a selfmonitoring study in individuals with obsessive-compulsive disorder. J Cogn Psychother. 2014;28(2):134-144.

31. Rachman S. A cognitive theory of compulsive checking. Behav Res Ther. 2002;40(6):625-639.

32. Coles ME, Sharkey KM. Compulsion or chronobiology? A case of severe obsessive-compulsive disorder treated with cognitivebehavioral therapy augmented with chronotherapy. J Clin Sleep Med. 2011;7(3):307-309.

33. Cameron OG, Lee MA, Kotun J, McPhee KM. Circadian symptom fluctuations in people with anxiety disorders. $J$ Affect Disord. 1986;11(3):213-218.

34. Harkin B, Kessler K. The role of working memory in compulsive checking and OCD: a systematic classification of 58 experimental findings. Clin Psychol Rev. 2011;31(6):1004-1021.

35. Harrison Y, Jones K, Waterhouse J. The influence of time awake and circadian rhythm upon performance on a frontal lobe task. Neuropsychologia. 2007;45(8):1966-1972.

36. Wright KP Jr, Hull JT, Czeisler CA. Relationship between alertness, performance, and body temperature in humans. Am J Physiol Regul Integr Comp Physiol. 2002;283(6):R1370-R1377.

37. de Vries FE, de Wit SJ, Cath DC, et al. Compensatory frontoparietal activity during working memory: an endophenotype of obsessivecompulsive disorder. Biol Psychiatry. 2014;76(11):878-887.

38. Vandewalle G, Archer SN, Wuillaume C, et al. Functional magnetic resonance imaging-assessed brain responses during an executive task depend on interaction of sleep homeostasis, circadian phase, and PER3 genotype. J Neurosci. 2009;29(25):7948-7956.

39. Insel TR, Murphy DL. The psychopharmacological treatment of obsessive-compulsive disorder: a review. J Clin Psychopharmacol. 1981;1(5):304-311.

40. Hesse S, Muller U, Lincke T, et al. Serotonin and dopamine transporter imaging in patients with obsessive-compulsive disorder. Psychiatry Res. 2005;140(1):63-72. 
41. Pogarell O, Hamann C, Popperl G, et al. Elevated brain serotonin transporter availability in patients with obsessive-compulsive disorder. Biol Psychiatry. 2003;54(12):1406-1413.

42. Hollander E, DeCaria C, Nitescu A, et al. Noradrenergic function in obsessive-compulsive disorder: behavioral and neuroendocrine responses to clonidine and comparison to healthy controls. Psychiatry Res. 1991;37(2):161-177.

43. Hollander E, DeCaria CM, Nitescu A, et al. Serotonergic function in obsessive-compulsive disorder. Behavioral and neuroendocrine responses to oral $\mathrm{m}$-chlorophenylpiperazine and fenfluramine in patients and healthy volunteers. Arch Gen Psychiatry. 1992;49(1):21-28.

44. Roman V, Walstra I, Luiten PG, Meerlo P. Too little sleep gradually desensitizes the serotonin 1 A receptor system. Sleep. 2005;28(12):1505-1510.

45. Piras F, Piras F, Chiapponi C, Girardi P, Caltagirone C, Spalletta G. Widespread structural brain changes in OCD: a systematic review of voxel-based morphometry studies. Cortex. 2015;62:89-108.

46. Baxter LR Jr, Phelps ME, Mazziotta JC, Guze BH, Schwartz JM, Selin CE. Local cerebral glucose metabolic rates in obsessive-compulsive disorder. A comparison with rates in unipolar depression and in normal controls. Arch Gen Psychiatry. 1987;44(3):211-218.

47. Swedo SE, Schapiro MB, Grady CL, et al. Cerebral glucose metabolism in childhood-onset obsessive-compulsive disorder. Arch Gen Psychiatry. 1989;46(6):518-523.

48. Machlin SR, Harris GJ,Pearlson GD, Hoehn-Saric R, Jeffery P, Camargo EE. Elevated medial-frontal cerebral blood flow in obsessive-compulsive patients: a SPECT study. Am J Psychiatry. 1991;148(9):1240-1242.

49. Rauch SL, Jenike MA, Alpert NM, et al. Regional cerebral blood flow measured during symptom provocation in obsessive-compulsive disorder using oxygen 15-labeled carbon dioxide and positron emission tomography. Arch Gen Psychiatry. 1994;51(1):62-70.

50. Whiteside SP, Port JD, Abramowitz JS. A meta-analysis of functional neuroimaging in obsessive-compulsive disorder. Psychiatry Res. 2004;132(1):69-79.

51. Menzies L, Chamberlain SR, Laird AR, Thelen SM, Sahakian BJ, Bullmore ET. Integrating evidence from neuroimaging and neuropsychological studies of obsessive-compulsive disorder: the orbitofronto-striatal model revisited. Neurosci Biobehav Rev. 2008;32(3):525-549.

52. Maia TV, Cooney RE, Peterson BS. The neural bases of obsessivecompulsive disorder in children and adults. Dev Psychopathol. 2008;20(4):1251-1283.

53. Via E, Cardoner N, Pujol J, et al. Amygdala activation and symptom dimensions in obsessive-compulsive disorder. $\mathrm{Br} J$ Psychiatry. 2014;204(1):61-68.

54. Saxena S, Brody AL, Schwartz JM, Baxter LR. Neuroimaging and frontal-subcortical circuitry in obsessive-compulsive disorder. $\mathrm{Br} J$ Psychiatry Suppl. 1998;(35):26-37.

55. Nakao T, Okada K, Kanba S. Neurobiological model of obsessivecompulsive disorder: evidence from recent neuropsychological and neuroimaging findings. Psychiatry Clin Neurosci. 2014;68(8): 587-605.

56. Simon D, Adler N, Kaufmann C, Kathmann N. Amygdala hyperactivation during symptom provocation in obsessive-compulsive disorder and its modulation by distraction. Neuroimage Clin. 2014;4:549-557.

57. Jenike MA, Baer L, Minichiello M, editors. Obsessive-Compulsive Disorders: Practical Management. St Louis, MO: Mosby; 1998.

58. Figee M, Vink M, de Geus F, et al. Dysfunctional reward circuitry in obsessive-compulsive disorder. Biol Psychiatry. 2011;69(9):867-874.

59. Jung WH, Kang DH, Han JY, et al. Aberrant ventral striatal responses during incentive processing in unmedicated patients with obsessivecompulsive disorder. Acta Psychiatr Scand. 2011;123(5):376-386.

60. Murray G, Nicholas CL, Kleiman J, et al. Nature's clocks and human mood: the circadian system modulates reward motivation. Emotion. 2009;9(5):705-716.

61. Hasler BP, Mehl MR, Bootzin RR, Vazire S. Preliminary evidence of diurnal rhythms in everyday behaviors associated with positive affect. J Res Pers. 2008;42(6):1537-1546.
62. Sleipness EP, Sorg BA, Jansen HT. Diurnal differences in dopamine transporter and tyrosine hydroxylase levels in rat brain: dependence on the suprachiasmatic nucleus. Brain Res. 2007;1129(1):34-42.

63. Albrecht U. The circadian clock, reward, and memory. Front Mol Neurosci. 2011;4:41.

64. Hasler BP, Clark DB. Circadian misalignment, reward-related brain function, and adolescent alcohol involvement. Alcohol Clin Exp Res. 2013;37(4):558-565.

65. Karatsoreos IN, Bhagat S, Bloss EB, Morrison JH, McEwen BS. Disruption of circadian clocks has ramifications for metabolism, brain, and behavior. Proc Natl Acad Sci U S A. 2011;108(4):1657-1662.

66. Wu JC, Gillin JC, Buchsbaum MS, et al. Frontal lobe metabolic decreases with sleep deprivation not totally reversed by recovery sleep. Neuropsychopharmacology. 2006;31(12):2783-2792.

67. Goel N, Rao H, Durmer JS, Dinges DF. Neurocognitive consequences of sleep deprivation. Semin Neurol. 2009;29(4):320-339.

68. St-Onge MP, McReynolds A, Trivedi ZB, Roberts AL, Sy M, Hirsch J. Sleep restriction leads to increased activation of brain regions sensitive to food stimuli. Am J Clin Nutr. 2012;95(4):818-824.

69. Gujar N, Yoo SS, Hu P, Walker MP. Sleep deprivation amplifies reactivity of brain reward networks, biasing the appraisal of positive emotional experiences. J Neurosci. 2011;31(12):4466-4474.

70. van Grootheest DS, Cath DC, Beekman AT, Boomsma DI. Twin studies on obsessive-compulsive disorder: a review. Twin Res Hum Genet. 2005;8(5):450-458.

71. Hettema JM, Neale MC, Kendler KS. A review and meta-analysis of the genetic epidemiology of anxiety disorders. Am J Psychiatry. 2001;158(10):1568-1578

72. Stewart SE, Pauls DL. The genetics of obsessive-compulsive disorder. Focus. 2010;8(3):350-357.

73. Webb IC, Lehman MN, Coolen LM. Diurnal and circadian regulation of reward-related neurophysiology and behavior. Physiol Behav. 2015;143:58-69.

74. Forbes EE, Dahl RE, Almeida JR, et al. PER2 rs2304672 polymorphism moderates circadian-relevant reward circuitry activity in adolescents. Biol Psychiatry. 2012;71(5):451-457.

75. da Rocha FF, Malloy-Diniz L, Lage NV, Correa H. The relationship between the Met allele of the BDNF Val66Met polymorphism and impairments in decision making under ambiguity in patients with obsessivecompulsive disorder. Genes Brain Behav. 2011;10(5):523-529.

76. Vinberg M, Trajkovska V, Bennike B, Knorr U, Knudsen GM, Kessing LV. The BDNF Val66Met polymorphism: relation to familiar risk of affective disorder, BDNF levels and salivary cortisol. Psychoneuroendocrinology. 2009;34(9):1380-1389.

77. Yu H, Wang DD, Wang Y, Liu T, Lee FS, Chen ZY. Variant brain-derived neurotrophic factor Val66Met polymorphism alters vulnerability to stress and response to antidepressants. J Neurosci. 2012;32(12):4092-4101.

78. Bachmann V, Klein C, Bodenmann S, et al. The BDNF Val66Met polymorphism modulates sleep intensity: EEG frequency- and statespecificity. Sleep. 2012;35(3):335-344.

79. Moller-Levet CS, Archer SN, Bucca G, et al. Effects of insufficient sleep on circadian rhythmicity and expression amplitude of the human blood transcriptome. Proc Natl Acad Sci U S A. 2013;110(12):E1132-E1141.

80. Archer SN, Laing EE, Moller-Levet CS, et al. Mistimed sleep disrupts circadian regulation of the human transcriptome. Proc Natl Acad Sci US A. 2014;111(6):E682-E691.

81. Fineberg NA, Reghunandanan S, Simpson HB, et al. Obsessivecompulsive disorder (OCD): practical strategies for pharmacological and somatic treatment in adults. Psychiatry Res. 2015;227(1): 114-125.

82. McKay D, Sookman D, Neziroglu F, et al. Efficacy of cognitivebehavioral therapy for obsessive-compulsive disorder. Psychiatry Res. 2015;227(1):104-113.

83. Stanley MA, Turnei SM. Current status of pharmacological and behavioral treatment of obsessive-compulsive disorder. Behav Ther. 1995;26:163-186. 
84. Soomro GM, Altman D, Rajagopal S, Oakley-Browne M. Selective serotonin re-uptake inhibitors (SSRIs) versus placebo for obsessive compulsive disorder (OCD). Cochrane Database Syst Rev. 2008;(1):CD001765.

85. Bandelow B, Zohar J, Hollander E, et al. World Federation of Societies of Biological Psychiatry (WFSBP) guidelines for the pharmacological treatment of anxiety, obsessive-compulsive and post-traumatic stress disorders-first revision. World J Biol Psychiatry. 2008;9(4):248-312.

86. Piccinelli M, Pini S, Bellantuono C, Wilkinson G. Efficacy of drug treatment in obsessive-compulsive disorder. A meta-analytic review. Br J Psychiatry. 1995;166(4):424-443.

87. Greist JH, Jefferson JW, Kobak KA, Katzelnick DJ, Serlin RC. Efficacy and tolerability of serotonin transport inhibitors in obsessive-compulsive disorder. A meta-analysis. Arch Gen Psychiatry. 1995;52(1):53-60.

88. Ackerman DL, Greenland S. Multivariate meta-analysis of controlled drug studies for obsessive-compulsive disorder. J Clin Psychopharmacol. 2002;22(3):309-317.

89. Pigott TA, Seay SM. A review of the efficacy of selective serotonin reuptake inhibitors in obsessive-compulsive disorder. J Clin Psychiatry. 1999;60(2):101-106.

90. Albelda N, Joel D. Animal models of obsessive-compulsive disorder: exploring pharmacology and neural substrates. Neurosci Biobehav Rev. 2012;36(1):47-63.

91. Lopes AC, de Mathis ME, Canteras MM, Salvajoli JV, Del Porto JA, Miguel EC. Atualização sobre o tratamento neurocirúrgico do transtorno obsessivo-compulsivo. [Update on neurosurgical treatment for obsessive compulsive disorder]. Rev Bras Psiquiatr. 2004;26(1):62-66. Portuguese.

92. Burguiere E, Monteiro P, Mallet L, Feng G, Graybiel AM. Striatal circuits, habits, and implications for obsessive-compulsive disorder. Curr Opin Neurobiol. 2015;30:59-65.

93. Goodman WK, Ward HE, Murphy TK. Biologic approaches to treatment-refractory obsessive-compulsive disorder. Psychiatr Ann. 1998;28(11):641

94. Paino M, Fonseca-Pedrero E, Bousoño M, Lemos-Giráldez S. Lighttherapy applications for DSM-IV-TR disease entities. Eur J Psychiatry. 2009;23(3):166-176.

95. Brinkhuijsen M, Koenegracht F, Meesters Y. Symptoms of seasonal affective disorder and of obsessive-compulsive disorder reduced by light therapy. J Affect Disord. 2003;74(3):307-308.

96. Hoflich G, Kasper S, Moller HJ. Erfolgreiche Behandlung eines saisonalen Zwangssyndroms mit Lichttherapie. [Light therapy in the treatment of a seasonal form of obsessive-compulsive disorder]. Nervenarzt. 1992;63(11):701-704. German.

97. Yoney TH, Pigott TA, L'Heureux F, Rosenthal NE. Seasonal variation in obsessive-compulsive disorder: preliminary experience with light treatment. Am J Psychiatry. 1991;148(12):1727-1729.

98. Partonen T, Lonnqvist J. Seasonal affective disorder. Lancet. 1998;352(9137):1369-1374.

99. Terman M, Lewy AJ, Dijk DJ, Boulos Z, Eastman CI, Campbell SS. Light treatment for sleep disorders: consensus report. IV. Sleep phase and duration disturbances. J Biol Rhythms. 1995;10(2):135-147.

100. Amir S, Robinson B, Ratovitski T, Rea MA, Stewart J, Simantov R. A role for serotonin in the circadian system revealed by the distribution of serotonin transporter and light-induced Fos immunoreactivity in the suprachiasmatic nucleus and intergeniculate leaflet. Neuroscience. 1998;84(4):1059-1073

101. Klein DC, Weller JL. Rapid light-induced decrease in pineal serotonin $\mathrm{N}$-acetyltransferase activity. Science. 1972;177(4048):532-533.

102. Cuesta M, Mendoza J, Clesse D, Pevet P, Challet E. Serotonergic activation potentiates light resetting of the main circadian clock and alters clock gene expression in a diurnal rodent. Exp Neurol. 2008;210(2):501-513.

103. Knoch ME, Siegel D, Duncan MJ, Glass JD. Serotonergic mediation of constant light-potentiated nonphotic phase shifting of the circadian locomotor activity rhythm in Syrian hamsters. Am J Physiol Regul Integr Comp Physiol. 2006;291(1):R180-R188.
104. Kaur G, Thind R, Glass JD. Brief constant light accelerates serotonergic re-entrainment to large shifts of the daily light/dark cycle. Neuroscience. 2009;159(4):1430-1440.

105. de Bodinat C, Guardiola-Lemaitre B, Mocaer E, Renard P, Munoz C, Millan MJ. Agomelatine, the first melatonergic antidepressant: discovery, characterization and development. Nat Rev Drug Discov. 2010;9(8):628-642.

106. Pandi-Perumal SR, MoscovitchA, Srinivasan V, Spence DW, Cardinali DP, Brown GM. Bidirectional communication between sleep and circadian rhythms and its implications for depression: lessons from agomelatine. Prog Neurobiol. 2009;88(4):264-271.

107. Stein DJ, Picarel-Blanchot F, Kennedy SH. Efficacy of the novel antidepressant agomelatine for anxiety symptoms in major depression. Hum Psychopharmacol. 2013;28(2):151-159.

108. Perugi G, Quaranta G, Bucci N. The use of agomelatine in OCD: effects on the motivational aspects and dysregulated circadian rhythms. Expert Opin Investig Drugs. 2015;24(5):705-713.

109. De Berardis D, Serroni N, Marini S, et al. Agomelatine augmentation of escitalopram therapy in treatment-resistant obsessivecompulsive disorder: a case report. Case Rep Psychiatry. 2012;2012: 642752.

110. da Rocha FF, Correa H. Is circadian rhythm disruption important in obsessive-compulsive disorder (OCD)? A case of successful augmentation with agomelatine for the treatment of OCD. Clin Neuropharmacol. 2011;34(4):139-140.

111. Signorelli MS, Concerto C, Battaglia E, Costanzo MC, Battaglia F, Aguglia E. Venlafaxine augmentation with agomelatine in a patient with obsessive-compulsive disorder and suicidal behaviors. SAGE Open Med Case Rep. 2014;2:1-3. DOI: 10.1177/2050313X14561778.

112. Fornaro M. Switching from serotonin reuptake inhibitors to agomelatine in patients with refractory obsessive-compulsive disorder: a 3 month follow-up case series. Ann Gen Psychiatry. 2011; 10(1):5.

113. Tzavellas E, Karaiskos D, Ilias I, Liappas I, Paparrigopoulos T. Agomelatine augmentation in obsessive compulsive disorder: a preliminary report. Psychiatriki. 2014;25(3):179-184.

114. De Berardis D, Serroni N, Campanella D, et al. A case of obsessivecompulsive disorder successfully treated with agomelatine monotherapy. J Clin Psychopharmacol. 2012;32(2):289-290.

115. Antoniadis D, Floros GD, Nikolaidis N, Garyfallos G. Response to agomelatine: treatment of an obsessive skin picking episode. Ann Clin Psychiatry. 2013;25(3):228-229.

116. Fornaro M, Prestia D, Colicchio S, Perugi G. A systematic, updated review on the antidepressant agomelatine focusing on its melatonergic modulation. Curr Neuropharmacol. 2010;8(3):287-304.

117. Tardito D, Milanese M, Bonifacino T, et al. Blockade of stress-induced increase of glutamate release in the rat prefrontal/frontal cortex by agomelatine involves synergy between melatonergic and 5-HT2C receptor-dependent pathways. BMC Neurosci. 2010;11:68.

118. Weitzman ED, Czeisler CA, Coleman RM, et al. Delayed sleep phase syndrome. A chronobiological disorder with sleep-onset insomnia. Arch Gen Psychiatry. 1981;38(7):737-746.

119. Trauer JM, Qian MY, Doyle JS, Rajaratnam SM, Cunnington D. Cognitive behavioral therapy for chronic insomnia: a systematic review and meta-analysis. Ann Intern Med. 2015;163(3):191-204.

120. Taylor DJ, Pruiksma KE. Cognitive and behavioural therapy for insomnia (CBT-I) in psychiatric populations: a systematic review. Int Rev Psychiatry. 2014;26(2):205-213.

121. Grandin LD, Alloy LB, Abramson LY. The social zeitgeber theory, circadian rhythms, and mood disorders: review and evaluation. Clin Psychol Rev. 2006;26(6):679-694.

122. Bouwkamp CG, de Kruiff ME, van Troost TM, et al. Interpersonal and social rhythm group therapy for patients with bipolar disorder. Int J Group Psychother. 2013;63(1):97-115.

123. Frank E, Kupfer DJ, Thase ME, et al. Two-year outcomes for interpersonal and social rhythm therapy in individuals with bipolar I disorder. Arch Gen Psychiatry. 2005;62(9):996-1004. 
124. Perugi G, Akiskal HS, Pfanner C, et al. The clinical impact of bipolar and unipolar affective comorbidity on obsessive-compulsive disorder. J Affect Disord. 1997;46(1):15-23.

125. Perugi G, Toni C, Frare F, Travierso MC, Hantouche E, Akiskal HS. Obsessive-compulsive-bipolar comorbidity: a systematic exploration of clinical features and treatment outcome. J Clin Psychiatry. 2002;63(12):1129-1134.
126. Tukel R, Meteris H, Koyuncu A, Tecer A, Yazici O. The clinical impact of mood disorder comorbidity on obsessive-compulsive disorder. Eur Arch Psychiatry Clin Neurosci. 2006;256(4):240-245.

127. Hantouche EG, Angst J, Demonfaucon C, Perugi G, Lancrenon S, Akiskal HS. Cyclothymic OCD: a distinct form? J Affect Disord. 2003;75(1):1-10.

\section{Publish your work in this journal}

ChronoPhysiology and Therapy is an international, peer-reviewed, open access journal focusing on research into the cyclic variations and rhythmicity in physiological processes in the body and the research and development and optimal timing of administration of therapeutic targets to achieve improved outcomes and quality of life for the patient. The
Dovepress

manuscript management system is completely online and includes a very quick and fair peer-review system. Visit http://www.dovepress.com testimonials.php to read real quotes from published authors. 